\title{
Chemical Ordering in In-Sn Liquid Alloy
}

\author{
D. Adhikari \\ Mahendra Morang Adarsh Multiple Campus,Tribhuvan University, Biratnagar \\ e-mail:adksbdev@yahoo.com
}

\begin{abstract}
Quasi-chemical expression has been used for weakly interacting compound forming binary alloy to obtain energy parameters and their temperature derivative for In-Sn liquid alloy at $700 \mathrm{~K}$. These energy parameters have then been used to calculate thermodynamic functions, such as free energy of mixing, heat of mixing, entropy of mixing and microscopic functions, such as concentration fluctuation in long wavelength limit, Warren-Cowely short range order parameter. The analysis reveals that the energy parameters are temperature dependent and the In-Sn liquid alloy at $700 \mathrm{~K}$ is weakly interacting heterocoordination system. The observed thermodynamic properties of In-Sn alloy in molten state have been successfully explained by assuming $\operatorname{In}_{3} \mathrm{Sn}$ complex on the basis of the quasichemical formalism for weakly interacting system.
\end{abstract}

Key words: In-Sn alloys, short- range order parameter, structural properties

\section{Introduction}

The determination of thermodynamic and structural properties of mixing of binary liquid alloys is one of the most intense research fields in metallurgical science. Thermodynamic and structural properties of the initial melt play an important role in the formation of solid alloy. However, it is much more difficult to understand the properties of disordered systems, such as liquid alloy than that of crystals. Therefore several models have long been employed by the theoreticians (Flory 1942, Jordan 1972, Bhatia \& Hargrove 1974, lele \& Rao 1981, Bhatia \& Singh 1984, Sommer 1990, Young 1992, Adhikari et al. 2010) to solve the complexities of explaining the alloying behaviour of binary liquid alloys. In present work, quasi-chemical expression for weakly interacting system (Singh 1987) has been employed to describe the thermodynamic and structural properties of In-Sn liquid alloy.

The study of lead-free solder is very important in electronic industry. The commonly used solder consists of lead which is poisonous to human and creates several environmental problems. The In-Sn alloy system is one of the foundry alloys for low melting lead-free solders. Such alloys can exhibit considerable viscoelasticity and the effective performance of these solders in electronic devices is related to their viscoelastic behaviour. This aspect of In-Sn alloy led the authors to its thermodynamic investigation. In addition, thermodynamic calculation has proven to be an important tool in the design and understanding of new lead-free solder alloys.

The phase diagram shows the existence of $\beta$ - $\operatorname{In}_{3} \mathrm{Sn}$ and ã-ãIn ${ }_{4} \mathrm{Sn}$ intermediate phase in the liquid state of In-Sn. We have studied both of these phases and concluded that $\beta$ - $\operatorname{In}_{3} \mathrm{Sn}$ is energetically favoured in molten In-Sn system. The theoretical formalism is provided in Section 2 and Section 3 deals with the numerical results and discussion. A conclusion is provided in section 4 .

\section{Methodology}

The existence of preferential association $A_{\mu} B_{v}$ among the constituent species in the liquid state of In-Sn alloy ( where $A \equiv I n, B \equiv S n, \mu=3$ and $v=1$ ) has been assumed. Thus the binary alloys can be considered as ternary mixture of A atoms, B atoms and a number of complexes. Bhatia and Singh (Bhatia \& 
Singh 1984) developed quasi-chemical model assuming the energy of an $\mathrm{AB}, \mathrm{AA}$, or $\mathrm{BB}$ bond depends on whether that bond is part of the complex or not. In quasi-chemical model, the thermodynamic properties are obtained by solving the grand partition function with the consideration that the energy of a given nearest bond is different if it belongs to the complex than if it does not. The excess free energy of mixing in terms of the ratio of the two activity coefficients,

$\gamma=\frac{\gamma_{\mathrm{A}}}{\gamma_{\mathrm{B}}}$ as

$\mathrm{G}=1 \mathrm{~N} / \mathrm{Nk} \mathrm{B}_{\mathrm{B}} \mathrm{T}=\int_{0}^{c} \ln \gamma \mathrm{dc}$

where $\gamma_{A}$ and $\gamma_{B}$ are respective activity coefficients of constituents $A$ and $B$ of the alloy $A$ $B N, k_{B}$ and $T$ are Avogardo's number, Boltzmann's constant and tem perature respectively and $c$ is concentration of A. Eq. (1) can be expressed in terms of probability that the bond in the cluster is part of the complex and can be solved in terms of ordering energies. A detail of the formulation is given in ref. (Singh 1987).

For all binary alloys in which intermetallic compounds are formed by weak interaction, the quasi- chemical expression for excess free energy of mixing ( $\left.\mathrm{G}_{\mathrm{II}}^{\pi}\right)$ can be written as (Singh 1987)

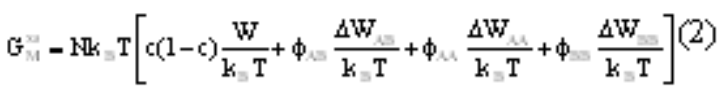
where $\phi_{i j}(i, j=A, B)$ are the simple polynomials in c depending on $\mu$ and $v$ values. W's are ordering energies. It may be noted that $W_{A A}=0$ if $\mu=1$ and $\mathrm{W}_{\mathrm{BB}}=0$ if $v=1$. F or $\mu=3$ and $v=1$ (e.g. $\mathrm{In}_{3} \mathrm{Sn}$ ) the values of $\phi$ 's are [18]:

$\phi_{A B}=\left[\frac{1}{5} c+\frac{2}{3} c^{3}-c^{4}-\frac{1}{5} c^{3}+\frac{1}{3} c^{6}\right]$

$\phi_{\mathrm{AA}}=\left[-\frac{3}{20} c+\frac{2}{3} c^{3}-\frac{3}{4} c^{4}+\frac{2}{5} c^{3}-\frac{1}{6} c^{6}\right]$

$\phi_{\mathrm{BB}}=0$

Using standard thermodynamic relations $\mathrm{S}_{\mathrm{WI}}^{\widetilde{w}}=-\left(\frac{\partial \mathrm{G}_{\mathrm{WI}}^{\tilde{u}}}{\partial \mathrm{T}}\right)_{\mathrm{p}}$ and Eq. (2) the ex cess entropy of mixing m ay be expressed as

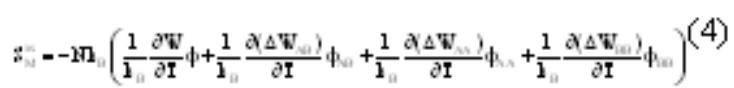

The heat of mixing $\mathrm{H}_{M}$ can be calculated using $E$ qs. (2) and (4) and standard therm odynamic relation,

$$
\mathrm{H}_{\mathrm{II}}=\mathrm{G}_{\mathrm{II}}^{\pi}+\mathrm{TS}_{\mathrm{II}}^{\pi}
$$

The long wave-length limit of the concentrationconcentration structure factor $\left(\mathrm{S}_{\mathrm{Cc}}(0)\right)$ is an important microscopic function (Singh 1987, Adrikari 2011) to understand the nature of atomic order in the binary liquid alloy. The $S_{C c}(0)$ can be expressed in terms of $\mathrm{G}_{M}$ as

$\mathrm{S}_{\mathrm{CC}}(0)=N \mathrm{Nk}_{\mathrm{H}} \mathrm{T}\left(\frac{\partial^{2} \mathrm{G}_{\mathrm{M}}}{\partial \mathrm{c}^{2}}\right)^{-1}$

$\mathrm{U}$ sing Eqs. (2) and (6), we get $\mathrm{S} C \mathrm{C}(0)$

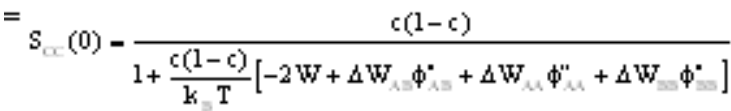

where $\phi_{i \mathrm{j}}^{\prime \prime}=\frac{\partial^{2} \phi_{i j}}{\partial c^{2}}(i, j=A, B)$. At a given composition, if $\operatorname{Scc}(0)<\mathrm{S}_{\mathrm{CC}}^{\mathrm{id}}(0)$, ordering in liquid alloy is expected and if $\operatorname{Scc}(0)>\mathrm{S}$ CC $(0)$, there is tendency of segregation.

The warren-Cowley short-range order parameter $\left({ }_{1}\right)$, which is an important parameter to quantify the degree of atomic order in the molten alloys, can be estimated from the knowledge of $\mathrm{S}_{\mathrm{CC}}(0)$ as

$$
\text { where } S=\frac{S_{C C}(0)}{S_{C C}^{\text {id }}(0)}
$$

$\mathrm{S}_{\mathrm{CC}}^{\mathrm{id}}(0)=\mathrm{c}(1-\mathrm{c})$ and $\mathrm{z}$ is co-ordination number. $\mathrm{z}$

$=10$ has been taken for our calculation. We note that varying the value of $z$ does not have any effect on the position of the minima of $\alpha_{1}$; the effect is to vary the depth while the overall feature remains unchanged. The knowledge of á- ${ }_{1}$ is very useful to provide an immediate insight into the nature of the local arrangements of atoms in the mixture.

\section{Results and Discussion}

The best fit values of energy parameters for the calculation of free energy of mixing of In-Sn alloy in molten state at $700 \mathrm{~K}$ using Eq. (2) are found to be

$\frac{W}{k_{B} T}=-0.22, \quad \frac{\Delta W_{A B}}{k_{B} T}=-0.60$, 


$$
\frac{\Delta \mathrm{W}_{\mathrm{AA}}}{\mathrm{k}_{\mathrm{B}} \mathrm{T}}=-0.21, \quad \frac{\Delta \mathrm{W}_{\mathrm{BB}}}{\mathrm{k}_{\mathrm{B}} \mathrm{T}}=0
$$

The negative values of energy parameters are the indicators of the presence of hetero-pairs, i.e. In-Sn, as the nearest neighbours in the alloy. The observed free energy of mixing of In-Sn alloy in molten state at $700 \mathrm{~K}$ (Hultgren et al. 1973) is symmetrical at equiatomic composition, $\mathrm{c}=1 / 2$. The theoretical analysis also shows that the free energy of mixing of this alloy is symmetrical at equiatomic composition. The theoretical and experimental values of $\mathrm{G}_{\mathrm{M}}$ are in a very good agreement (Fig.1).

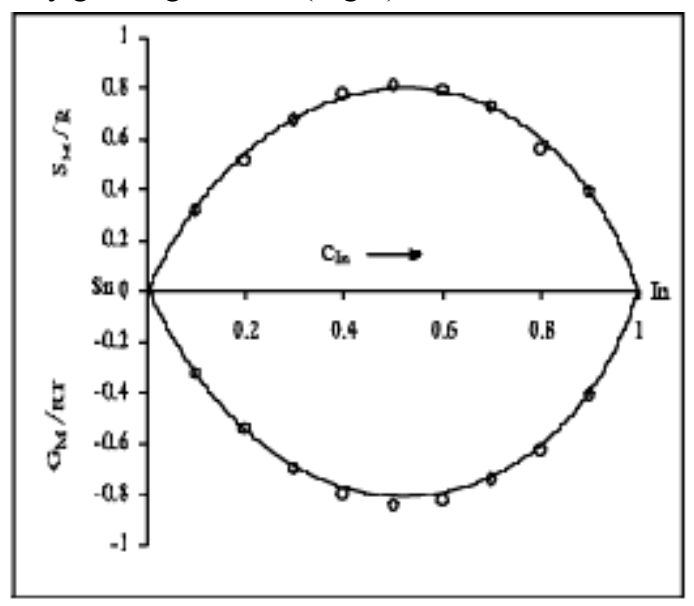

Fig 1

Fig. 1. Upper part: Entropy of mixing $\left(\mathrm{S}_{\mathrm{M}} / \mathrm{R}\right)$ versus $\mathrm{c}_{\mathrm{In}}$, Lower part: Free energy of mixing $\left(\mathrm{G}_{\mathrm{M}} / \mathrm{RT}\right)$ versus $\mathrm{c}_{\mathrm{In}}$ of liquid In-Sn solution $(700 \mathrm{~K})$; (---)

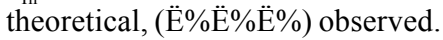

It is observed that if the energy parameters are supposed to be independent of temperature, i.e., $\frac{\partial W}{\partial T}=0$, then $\mathrm{H}_{\mathbf{M}}$ and $\mathrm{S}_{\mathbf{W}}$ so obtained are in very poor agreement with experimental data. This simply suggests importance of the dependence of interaction energies on temperature. The best fit values of the temperature derivative of energy parameters for the formation complex In $\mathrm{Sn}$ are found to be

$$
\begin{gathered}
\frac{1}{\mathrm{k}_{\mathrm{B}}} \frac{\partial \mathrm{W}}{\partial \mathrm{T}}=-0.20, \frac{1}{\mathrm{k}_{\mathrm{B}}} \frac{\partial \Delta \mathrm{W}_{\mathrm{AB}}}{\partial \mathrm{T}}=-0.60, \\
\frac{1}{\mathrm{k}_{\mathrm{B}}} \frac{\partial \Delta \mathrm{W}_{\mathrm{AA}}}{\partial \mathrm{T}}=-0.60, \frac{1}{\mathrm{k}_{\mathrm{B}}} \frac{\partial \Delta \mathrm{W}_{\mathrm{BB}}}{\partial \mathrm{T}}=0
\end{gathered}
$$

Using above temperature dependent parameters in Eqs. (4) and (5) entropy of mixing and heat of mixing of InSn alloys in liquid state at $700 \mathrm{~K}$ have bee calculated. The calculated and experimental values of $\mathrm{H}_{\mathbf{M}}$ and $S_{\mathrm{WI}}$ are plotted against concentration of In in Figs. 1 and 2 respectively. The observed values of $\mathrm{S}_{\mathrm{M}}$ (Hultgren et al. 1973) is symmetrical around $\mathrm{c}=1 / 2$. The calculated values confirm the observed nature of $\mathrm{S}_{\mathrm{M}}$. The calculated and observed values of $\mathrm{S}_{\mathrm{M}}$ are in a good agreement (Fig.1).

The values of heat of mixing of In-Sn liquid alloy at 700 $\mathrm{K}$ are very small negative values. A discrepancy may be noticed in the observed and calculated values of $\mathrm{H}_{\mathrm{M}}$ in Fig.2. This discrepancy may be due to the neglect of $\Delta W_{\mathrm{BB}}$, its temperature derivative and vibrational energy. Moreover, more than one complexe may form in the liquid state which are not considered in our analysis.

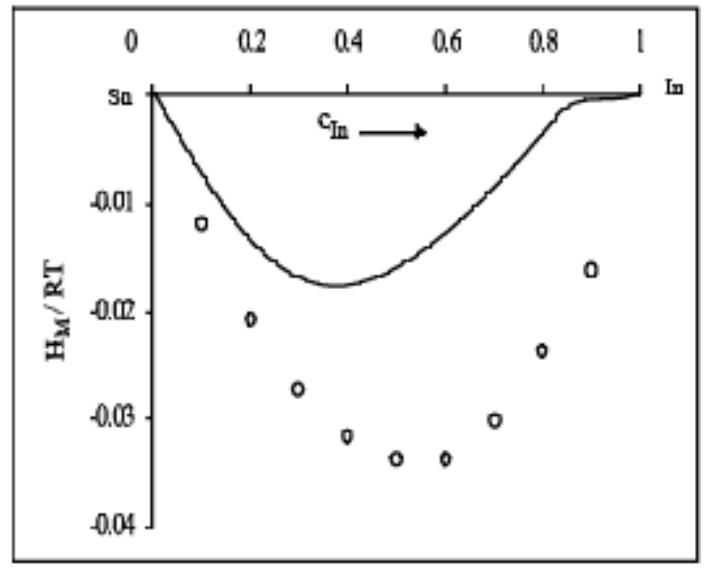

Fig. 2

Fig. 2. Heat of mixing $\left(\mathrm{H}_{\mathrm{KI}} / \mathrm{RT}\right)$ versus $\mathrm{c}_{\mathrm{h}}$ of liquid $\mathrm{In}-\mathrm{Sn}$ solution (700 K); ( $\longrightarrow$ ) theoretical, (0oo) observed.

Eq. (7) has been used to compute $\mathrm{S}_{\mathrm{CC}}(0)$ for In-Sn liquid alloy at $700 \mathrm{~K}$. The basic inputs for the computation of $\mathrm{S}_{\mathrm{CC}}(0)$ are energy parameters. The consistency is maintained by using the same values of energy parameters which were used for the evaluation of $\mathrm{G}_{\mathrm{M}}, \mathrm{S}_{\mathrm{M}}$ and $\mathrm{H}_{\mathrm{M}}$. It is observed that the calculated values of $\mathrm{S}_{\mathrm{CC}}(0)$ is less that ideal value $\left(\mathrm{S}_{\mathrm{cc}}^{\mathrm{il}}(0)\right)$ at whole range of concentration. This simply suggests that there is a tendency of complex formation in In-Sn liquid alloy at $700 \mathrm{~K}$ in whole range of 
Nepal Journal of Science and Technology Vol. 14, No. 2 (2013) 161-164

concentration. But this tendency is very weak as the deviation of $\mathrm{S}_{\mathrm{CC}}(0)$ is not large in comparison of the ideal value. However the deviation of $\mathrm{S}_{\mathrm{CC}}(0)$ from the ideal value is maximum near compound forming concentration, $\mathrm{c}=0.66$.

To analyse the degree of atomic ordering in molten In-Sn alloy, the Warren-Cowely short range ordering parameter, has been calculated. The knowledge of $\alpha_{1}$ provides an immediate insight into the nature of the local arrangements of atoms in the mixture. At equiatomic composition, one has $-1 \leq \alpha_{1} \leq 1$. The minimum possible value of $\alpha_{1}$ is $\alpha_{1}^{\min }=-1$ and that implies complete ordering of unlike atoms paring at nearest neighbours. On the other hand the maximum value of $\alpha_{1}$ is $\alpha_{1}^{\max }=+1$ which implies total segregation leading to the phase separation and $\alpha_{1}=0$ corresponds to a random distribution of atoms. It is observed that is negative throughout the whole range of concentration (Fig.3). The negative value of is the confirmation of heterocoordination in In-Sn liquid alloy as indicated by the result of $\mathrm{GM}, \mathrm{SCC}(0)$ and energy parameters. In addition, á $1>>-1$, and this suggests that there is a weak tendency of heteropairing between the atoms in the liquid alloy.

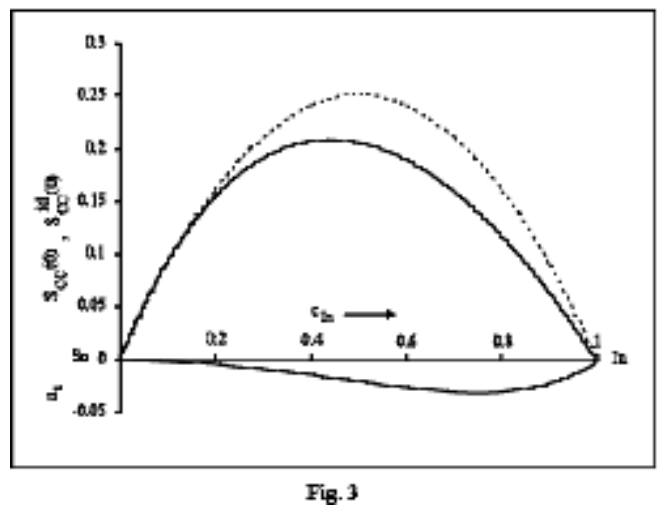

Fig. 3. Upper part: Concentration fluctuations in long wavelength limit $\left(\mathrm{S}_{\mathrm{cc}}(0)\right)$ versus $\mathrm{c}_{\mathrm{In}}$ of liquid In-Sn solution $(700 \mathrm{~K})$. Lower part: short range ordering parameter (á) of liquid In-Sn solution $(700 \mathrm{~K})$ versus $\mathrm{c}_{\mathrm{In}} ;(-)$ theoretical, $(-)$ ideal values.
Our theoretical analysis suggests that there is a tendency of unlike atoms pairing ( $\mathrm{In}-\mathrm{Sn}$ ) in liquid In-Sn alloys at all concentration. The analysis also shows that the molten In-Sn system is weakly interacting heterocoordination system. The energy parameters of this system also depend on temperature.

\section{Acknowledgements}

The author is grateful to Nepal Academy of Science and Technology, Nepal for providing financial support to pursue the research work.

\section{References}

Adhikari, D. 2011. Disorder in Cu-Pd liquid alloys. Phase Transitions 84: 308-314.

Adhikari, D., I. S. Jha, and B.P. Singh. 2010. Structural asymmetry in liquid $\mathrm{Fe}-\mathrm{Si}$ alloys. Philosophical Magazine 90: 2687-2694.

Bhatia, A.B. and R.N. Singh. 1984. A quasi-lattice theory compound forming molten alloys. Physics and Chemistry of Liquids 13(3): 177-190.

Bhatia, A.B. and W.H. Hargoove. 1974. Concentration fluctuation and thermodynamic properties of some compound forming binary molten alloys. Physical Review B10: 3186.

Flory, P.J. 1942. Thermodynamics of high polymer solutions. Journal of Chemical Physics 10: 51-61.

Hultgren, R., P.D. Desai, D.T. Hawkins, M. Gleiser, and K.K. Kelly. 1973. Selected values of the thermodynamic properties of binary alloys. ASM, Metal Park, p. 777.

Jordan, A.S. 1970. A theory of regular associated solution applied to the liquidus curves of the $\mathrm{Zn}-\mathrm{Te}$ and $\mathrm{Cd}-\mathrm{Te}$ system. Metallurgical Transactions 1: 239-249.

Lele, S. and P. Ramchandrarao. 1981. Estimation of complex concentration in a regular associated solution. Metallurgical Transactions 12 B: 659-666.

Singh, R.N. 1987. Short-range order and concentration fluctuations in binary molten alloys. Canadian Journal of Physics 65: 309.

Sommer, F. 1990. Thermodynamic properties of compound-forming liquid alloys. Journal of NonCrystalline Solids 117: 505-512.

Young, W.H. 1992. Structural and thermodynamic properties of NFE liquid metals and binary alloys. Rapidly Progress in Physics 55: 1769. 